\title{
Suicidality and aggression during antidepressant treatment: systematic review and meta-analyses based on clinical study reports
}

\author{
Tarang Sharma, , ,2 Louise Schow Guski,, ,2 Nanna Freund, , ,2 Peter C Gøtzsche' ,2
}

${ }^{1}$ Nordic Cochrane Centre,

Rigshospitalet, Copenhagen,

Denmark

2University of Copenhagen,

Faculty of Health and Medical

Sciences, Denmark

Correspondence to: T Sharma

Nordic Cochrane Centre,

Rigshospitalet, Blegdamsvej 9,

Department 7811, $2100 \varnothing$

Copenhagen, Denmark

ts@cochrane.dk

Additional material is published online only. To view please visit the journal online (http://dx.doi. org/10.1136/bmj.i65)

Cite this as: BMJ 2016;352:i65 http://dx.doi.org/10.1136/bmj.i65

Accepted: 03 December 2015

\section{ABSTRACT \\ OBJECTIVE \\ To study serious harms associated with selective serotonin and serotonin-norepinephrine reuptake inhibitors. \\ DESIGN \\ Systematic review and meta-analysis. \\ MAIN OUTCOME MEASURES \\ Mortality and suicidality. Secondary outcomes were} aggressive behaviour and akathisia.

\section{DATA SOURCES}

Clinical study reports for duloxetine, fluoxetine, paroxetine, sertraline, and venlafaxine obtained from the European and UK drug regulators, and summary trial reports for duloxetine and fluoxetine from Eli Lilly's website.

\section{ELIGIBILITY CRITERIA FOR STUDY SELECTION}

Double blind placebo controlled trials that contained any patient narratives or individual patient listings of harms.

\section{DATA EXTRACTION AND ANALYSIS}

Two researchers extracted data independently; the outcomes were meta-analysed by Peto's exact method (fixed effect model).

\section{RESULTS \\ We included 70 trials (64381 pages of clinical study reports) with 18526 patients. These trials had limitations in the study design and discrepancies in reporting, which may have led to serious under- reporting of harms. For example, some outcomes appeared only in individual patient listings in appendices, which we had for only 32 trials, and we did not have case report forms for any of the trials. Differences in mortality (all deaths were in adults, odds ratio $1.28,95 \%$ confidence interval 0.40 to 4.06 ),}

\section{WHAT IS ALREADY KNOWN ON THIS TOPIC}

Important information on harms is often missing in published trial reports

Clinical study reports should therefore be the preferred source for systematic reviews of drugs

Antidepressants can increase the risk of suicide in children and adolescents

\section{WHAT THIS STUDY ADDS}

Despite all the limitations we identified in the trials and in the clinical study reports, we found an increase in events of aggression with antidepressants (lost in adults alone), with a doubling of both suicidality and aggression in children and adolescents Selective reporting of relevant harms across the different sections of the clinical study reports meant that patient narratives, tables with individual patient listings (often found in appendices), and case report forms are needed for complete information Online summary reports of trials available from Eli Lilly's website are inadequate as source documents for identifying harms data suicidality (1.21, 0.84 to 1.74$)$, and akathisia $(2.04,0.93$ to 4.48) were not significant, whereas patients taking antidepressants displayed more aggressive behaviour $(1.93,1.26$ to 2.95$)$. For adults, the odds ratios were 0.81 (0.51 to 1.28) for suicidality, 1.09 (0.55 to 2.14) for aggression, and 2.00 ( 0.79 to 5.04 ) for akathisia. The corresponding values for children and adolescents were 2.39 (1.31 to 4.33), 2.79 (1.62 to 4.81), and 2.15 ( 0.48 to 9.65$)$. In the summary trial reports on Eli Lilly's website, almost all deaths were noted, but all suicidal ideation events were missing, and the information on the remaining outcomes was incomplete.

\section{CONCLUSIONS}

Because of the shortcomings identified and having only partial access to appendices with no access to case report forms, the harms could not be estimated accurately. In adults there was no significant increase in all four outcomes, but in children and adolescents the risk of suicidality and aggression doubled. To elucidate the harms reliably, access to anonymised individual patient data is needed.

\section{Introduction}

Selective serotonin reuptake inhibitors (SSRIs) and serotonin-norepinephrine reuptake inhibitors (SNRIs) are some of the most commonly prescribed drugs. ${ }^{12}$ SSRI induced suicidality was first reported in $1990^{3}$ but only became generally recognised after a BBC Panorama programme focused on it in 2002.4

A 2004 UK review showed a noticeable discrepancy between published and unpublished trials and increased suicidal behaviour in children and adolescents (aged $<18$ years), ${ }^{5}$ which resulted in serious warnings against these drugs being used in this age group. ${ }^{6}$ It is widely believed that the risk of suicide is not increased in adults, and support for this was provided by a Food and Drug Administration meta-analysis of about 100000 patients. $^{7}$ However, a large systematic review of published trials found an increase in suicide attempts with SSRI treatment, ${ }^{1}$ and another review using data submitted to the UK's Medicines and Healthcare products Regulatory Agency (MHRA) could not rule out an increased risk of suicidal behaviour during early treatment with these drugs. ${ }^{8}$

For aggressive behaviour (for example, hostility, assault) in general, reports are conflicting. ${ }^{9-15}$ A UK review using MHRA data found an increase in hostility in children and adolescents, ${ }^{16}$ and an analysis of adverse events reported to the FDA showed that antidepressants were disproportionately involved in cases of violence, including murder. ${ }^{17}$ Many cases of aggressive behaviour have been reported, ${ }^{24}$ but, unlike with 
suicidality, little systematic research has been undertaken. Perpetrators of school shootings and similar events have often been reported to be users of antidepressants ${ }^{18}$ and the courts have in many cases found them not guilty as a result of drug induced insanity. ${ }^{4}$

Akathisia is an extreme form of restlessness, which some patients describe as wanting to "jump out of their skin," that may increase the risk of suicide and vio-

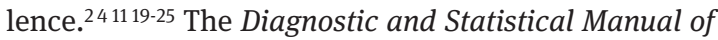
Mental Disorders describes akathisia or similar activation symptoms as "medication-induced movement disorder not otherwise specified." 26

Clinical study reports are detailed summaries of trial results prepared by the drug industry for submission to regulatory authorities to obtain authorisation for marketing. A recent review of clinical study reports showed that essential information on patient relevant outcomes was often missing in the published articles. ${ }^{27}$ Research undertaken by our centre using nine clinical study reports on duloxetine found that data on major harms was missing from journal articles and in summary trial reports. ${ }^{28}$ We did not have access to any case report forms (paper or electronic questionnaires that contain the collected data on each participant in the trial), although they would have been the ideal information source. ${ }^{28}$

We report here our results for mortality, suicidality, aggression, and akathisia based on clinical study reports for five different antidepressants.

\section{Methods}

In 2011, we requested clinical study reports on SSRIs and SNRIs from the European Medicines Agency and the UK's MHRA. We did not get access to clinical study reports for all trials or for all the commonly prescribed drugs, and we did not receive case report forms for any of the trials. One researcher (TS) selected those clinical study reports that described double blind placebo controlled trials and which contained patient narratives (brief summaries of deaths, serious adverse events, or other events of clinical importance) or listings of adverse events in individual patients (with details such as patient identifier, the adverse event (preferred term and verbatim term), duration, severity, and outcome). ${ }^{28}$

We were able to include five drugs: duloxetine, fluoxetine, paroxetine, sertraline, and venlafaxine (or venlafaxine extended release). We converted the clinical study reports to readable portable document format, and one researcher (TS) copied all relevant pages-with study information, protocols, all adverse event summaries and tables, relevant appendices (where available), patient narratives, and individual patient listings-for use in data extraction.

As a pilot, we randomly chose one report for each drug and read it in its entirety to help understand the different formats of the clinical study reports and to refine the data extraction form. We had planned that the second observer would extract the data blindly, with the treatment groups masked, but the pilot showed that the format and language used made blinding impossible. The primary researcher (TS) and a second observer (LSJ or NF) extracted data from the selected pages of all the clinical study reports independently; disagreements were resolved by discussion and documented using $\kappa$ statistics (see supplementary data A).

\section{Outcomes}

The primary outcomes were mortality and suicidality (suicide, suicide attempt or preparatory behaviour, intentional self harm, and suicidal ideation); secondary outcomes were aggressive behaviour and akathisia. To identify the primary outcomes, we used the same terms and phrases as those of the FDA $^{729}$ and added additional terms from our pilot. We searched the clinical study reports both electronically and manually. For people with more than one suicidality event, we counted only the most severe one, whereas this was not possible for the secondary outcomes, which only allowed us to count events. Terms for aggressive behaviour were informed by the pilot, and akathisia was identified by searching for "akathisia" in the text (see supplementary data A). All relevant events were classified using the Medical Dictionary for Regulatory Activities (MedDRA) coding dictionary. For duloxetine and fluoxetine, we compared the data with the summary trial reports from Eli Lilly's website. ${ }^{30}$

For meta-analysis of rare events, we reported odds ratios using Peto's exact method and calculated 95\% confidence intervals with a fixed effect model using RevMan 5.3.3132 All post-randomisation events were included, so when data from the lead-out and post-treatment phases were available, we combined them with the data from the randomised phase. In trials with multiple intervention arms, we added the data on arms arithmetically to get a combined drug arm. We planned and conducted subgroup analyses for adults for all outcomes and for suicides and suicide attempts combined, and did post-hoc analyses for suicides and children and adolescents and a sensitivity analysis removing data from fraudulent centres, as suggested by peer reviewers.

\section{Patient involvement}

No patients were involved in setting the research question or the outcome measures, nor were they involved in the design and implementation of the study. We plan to involve patient organisations in the dissemination of our results.

\section{Results}

We excluded 125 of the 198 clinical study reports: 96 were not double blind placebo controlled trials, 28 were studies in healthy volunteers, and one was a crossover trial (fig 1). Of the remaining 73 clinical study reports, we excluded five that had no patient narratives or individual patient listings of adverse events. The 68 included clinical study reports amounted to 64381 pages and corresponded to 70 trials.

\section{Trial characteristics and study design}

The experimental drugs were duloxetine (23 trials), fluoxetine $(n=3)$, paroxetine $(n=8)$, sertraline $(n=28)$, and venlafaxine $(n=8)$. In total, 10258 patients received a 
Clinical study reports available from regulators $(n=198)$

Excluded non-double blind randomised controlled trials, healthy volunteer studies, and crossover randomised controlled trials $(n=125)$

Total clinical study reports with double blind placebo controlled and/or active comparator randomised controlled trials $(n=73)$

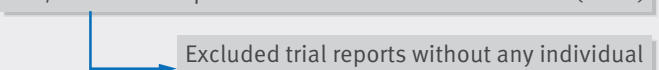
patient listings or narratives $(n=5)$

Total relevant randomised controlled trial clinical study reports included, corresponding to 70 randomised controlled trials $(n=68)$

Fig 1 | Flowchart showing selection of relevant studies for inclusion

drug and 6832 a placebo. Fifteen trials had an additional (SSRI or SNRI) comparator in 669 patients (228 receiving fluoxetine and 441 receiving paroxetine) and a tricyclic or tetracyclic comparator in 767 patients. Eleven of the trials ( $12 \%$ of the patients) concerned children and adolescents. Table 1 shows the indications for treatment; 34 trials included 7882 patients with major depressive disorder. Patients at risk of suicide were excluded in 44 trials (63\%); in 16 trials, suicide risk was not an exclusion criterion (23\%), whereas it was unknown in 10 trials (14\%). The randomised phase of the trials lasted from one to 54 weeks (median nine weeks).

Sixty trials (86\%) had a placebo lead-in period (4 to 14 days, median 7 days) and all of them excluded from randomisation those who improved while receiving placebo, as judged by their Hamilton scores or similar. Rarely was there any information about the numbers excluded.

It was unclear to what extent sedatives were allowed or used. Four duloxetine trials and four sertraline trials allowed benzodiazepines or similar psychoactive drugs. However, in at least 50 trials $(71 \%$, we did not have access to the full protocol for all the trials), sedatives such as choral hydrate or zolpidem were allowed if the patients had difficulty sleeping.

The quality of the clinical study reports varied. For 32 trials we had individual patient listings of adverse events for all patients (in appendices, apart from the venlafaxine trials where the listings were part of the main report). We had access to the protocol for 44 trials; for the remaining trials, only a summary of the study design was available. It seemed that all other appendices were either only "available on request" to the authorities or came under "the system of exceptions set out in the Regulation (EC) No 1049/2001," and so could not be released to us. This is in line with the guidance for clinical study reports, where certain appendices are not required to be submitted to the EMA. ${ }^{33}$ For 27 trials, we only had abbreviated or summary clinical study reports; some of these were titled accordingly whereas others were called clinical study reports, although they were only short summaries of about 100 pages. For four trials of sertraline, we only had summary reports combining two trials each (trials 51 and 52, and trials 53 and 54) for which the protocols were the same. We analysed the results accordingly. Key characteristics of the included trials are available in the supplementary data B.

The drug companies had concerns about the validity of the data or fraudulent behaviour in three trials. The data from one centre in trial 28 was not included in the efficacy analyses "due to concerns over the validity of the data," and in trial 34, one centre was shut down "following an internal audit that detected significant compliance violations.” Four centres in trial 70 exhibited potentially fraudulent behaviour: three centres had their study records "impounded by the Swiss police for fraud"; and for the fourth centre, "Many of the enrolled patients ... had identical evaluations for consecutive visits, and ... all 35 patients from this site had very similar evaluation patterns."

The interobserver agreement for our assessments was high ( $\kappa=0.94)$. Most disagreements resulted from errors in data extraction; discussion and consensus was needed for only two events.

\section{Mortality}

Sixteen deaths occurred, all in adults: one in the placebo lead-in phase and one in a 12 week lead-in phase during treatment with duloxetine $60 \mathrm{mg} /$ day. Post-randomisation, nine deaths occurred during treatment with an SSRI or SNRI and four with placebo (odds ratio $1.28,95 \%$ confidence interval 0.40 to 4.06 ) plus one with imipramine (table 2, fig 2, and supplementary data C). As none of the deaths occurred in fraudulent centres, no sensitivity analysis was needed.

Four deaths were misreported by the company, in all cases favouring the active drug. One death in a

\begin{tabular}{|c|c|}
\hline Indication & Drugs (No of trials) \\
\hline Major depressive disorder & $\begin{array}{l}\text { Duloxetine (12), fluoxetine (2), paroxetine (3), sertraline } \\
\text { (9), venlafaxine or venlafaxine extended release (8) }\end{array}$ \\
\hline Obsessive compulsive disorder & Fluoxetine (1), paroxetine (1), sertraline (7) \\
\hline Post-traumatic stress disorder & Paroxetine (3), sertraline (4) \\
\hline Stress urinary incontinence & Duloxetine (8) \\
\hline Generalised social phobia or social anxiety disorder or social phobia & Sertraline (2), paroxetine (1) \\
\hline Irritative symptoms of benign prostatic hyperplasia & Duloxetine (1) \\
\hline Diabetic peripheral neuropathic pain & Duloxetine (1) \\
\hline Fibromyalgia & Duloxetine (1) \\
\hline Non-insulin-dependent diabetes mellitus & Sertraline (1) \\
\hline
\end{tabular}




\begin{tabular}{|c|c|c|c|c|}
\hline \multirow[b]{2}{*}{ Phase of trial } & \multicolumn{4}{|l|}{ No of deaths } \\
\hline & $\begin{array}{l}\text { Before } \\
\text { randomisation }\end{array}$ & Drug arm & $\begin{array}{l}\text { Third arm } \\
\text { (imipramine) }\end{array}$ & $\begin{array}{l}\text { Placebo } \\
\text { arm }\end{array}$ \\
\hline Before randomisation & 2 & 0 & 0 & 0 \\
\hline Randomised phase & 0 & 8 & 1 & 3 \\
\hline Lead-out and post-treatment & 0 & 1 & 0 & 1 \\
\hline Total No of deaths & 2 & 9 & 1 & 4 \\
\hline
\end{tabular}

Drugs: duloxetine, fluoxetine, paroxetine, sertraline, venlafaxine.

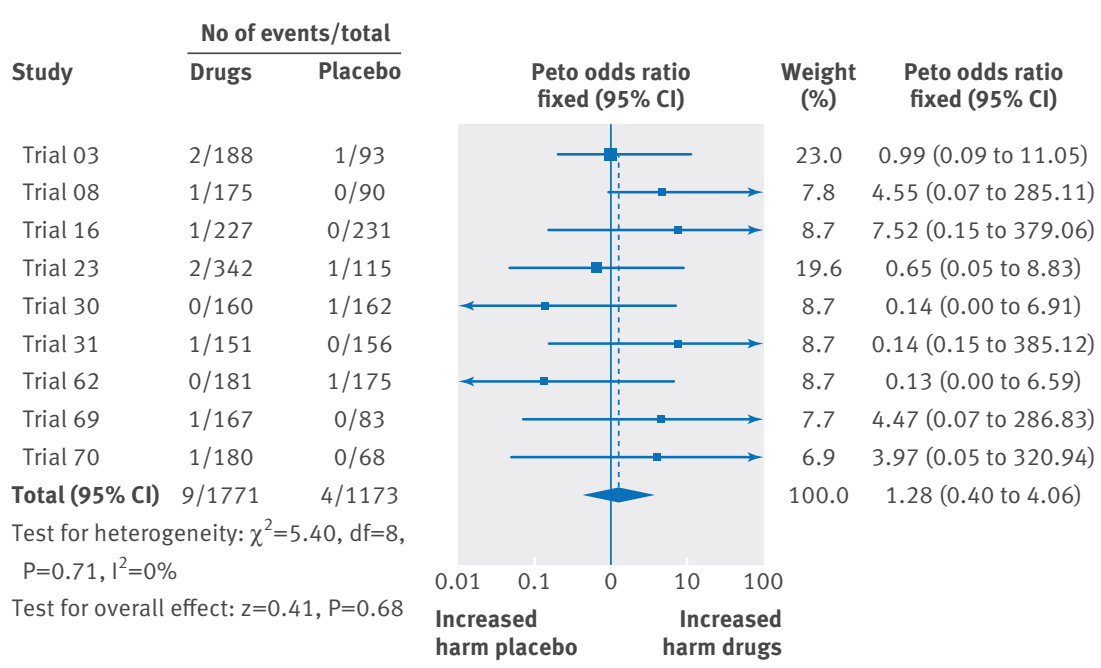

Fig 2 Meta-analysis of all cause mortality for selective serotonin reuptake inhibitors (SSRI s) or serotonin-norepinephrine reuptake inhibitors (SNRI s) compared with placebo postrandomisation

participant receiving paroxetine (trial 31) was called a post-study event, taking place 21 days after the patient had admitted to taking the last dose, but this was on day 63 out of the 84 days of randomised treatment. Moreover, the patient had detectable paroxetine in the blood at the time of death. A patient receiving venlafaxine (trial 69) attempted suicide by strangulation without forewarning and died five days later in hospital. Although the suicide attempt occurred on day 21 out of the 56 days of randomised treatment, the death was called a post-study event as it occurred in hospital and treatment had been discontinued because of the suicide attempt. Conversely, a patient receiving placebo (trial 62) died on day 404, 26 days after the randomised phase ended, but the death was not listed as a post-study event as the patient had allegedly taken treatment until the previous day. Finally, a death in a participant receiving venlafaxine (trial 70) that occurred three months after treatment was only noted in the patient narratives and nowhere else in the clinical study report.

\section{Suicidality}

Overall, 155 suicidality events took place, 13 before randomisation. The odds ratio post-randomisation for suicidality in patients was 1.21 (95\% confidence interval 0.84 to 1.74 ) and was similar for number of suicidality events $(1.14,0.80$ to 1.64$)$. The odds ratio for suicidality in adults was 0.81 (0.51 to 1.28) and 0.77 (0.49 to 1.21 for events) and for children and adolescents was 2.39 (1.31 to 4.33) and 2.24 (1.24 to 4.04 for events). None of the suicidality events occurred in patients from fraudulent centres. See table 3, fig 3 and supplementary data C and D.

\section{Suicides}

Six suicides were reported, one in the duloxetine lead-in phase. Post-randomisation five suicides were reported: two in the study drug group, two in the placebo group (odds ratio $0.58,95 \%$ confidence interval 0.07 to 4.48), and one in the imipramine group (see supplementary data $\mathrm{C}$ and $\mathrm{D}$ ).

\section{Suicide attempts}

We counted all attempted suicides, including intentional self harm (for example, slitting of wrists), intentional overdoses, and obvious preparatory events (for example, putting a knife to the wrist or neck, but being stopped before any harm). Six of the 73 events ( $n=70$ patients) took place before randomisation (four in participants taking duloxetine and two in participants taking placebo).

One of the events, in a participant taking placebo before randomisation, occurred on day 29, although the lead-in phase was supposed to last only 14 days. Also, one of the four suicide attempts in participants taking duloxetine before randomisation was only identified by

\begin{tabular}{|c|c|c|c|c|c|c|c|c|}
\hline Suicidality events & Duloxetine & Fluoxetine & Paroxetine & Sertraline & Venlafaxine & All drugs & Placebo & Imipramine \\
\hline \multicolumn{9}{|l|}{ Before randomisation } \\
\hline \multicolumn{9}{|l|}{ Drug event: } \\
\hline Suicides & 1 & - $^{\star}$ & $-^{\star}$ & $-{ }^{*}$ & $-^{*}$ & 1 & 0 & $-^{\star}$ \\
\hline Suicide attempts & 4 & $-^{*}$ & $-^{\star}$ & $-{ }^{\star}$ & $-^{*}$ & 4 & 2 & $-^{*}$ \\
\hline Suicidal ideation & 4 & $-^{\star}$ & $-^{\star}$ & $-{ }^{\star}$ & $-^{\star}$ & 4 & 2 & $-^{\star}$ \\
\hline Suicidality & 9 & & & & & 9 & 4 & \\
\hline \multicolumn{9}{|l|}{ Post-randomisation } \\
\hline \multicolumn{9}{|c|}{ Drug (any arm) event: } \\
\hline Suicides & 1 & 0 & 0 & 0 & 1 & 2 & 2 & 1 \\
\hline Suicide attempts & 8 & 5 & 18 & 9 & 3 & 43 & 22 & 2 \\
\hline Suicidal ideation & 8 & 1 & 18 & 11 & 3 & 41 & 25 & 4 \\
\hline Suicidality & 17 & 6 & 36 & 20 & 7 & 86 in 85 patients & 49 in 46 patients & 7 in 7 patients \\
\hline Total population & 4277 & 456 & 1766 & 3165 & 1263 & 10927 & 6832 & 767 \\
\hline
\end{tabular}

\footnotetext{
*No patients received these drugs pre-randomisation.
} 


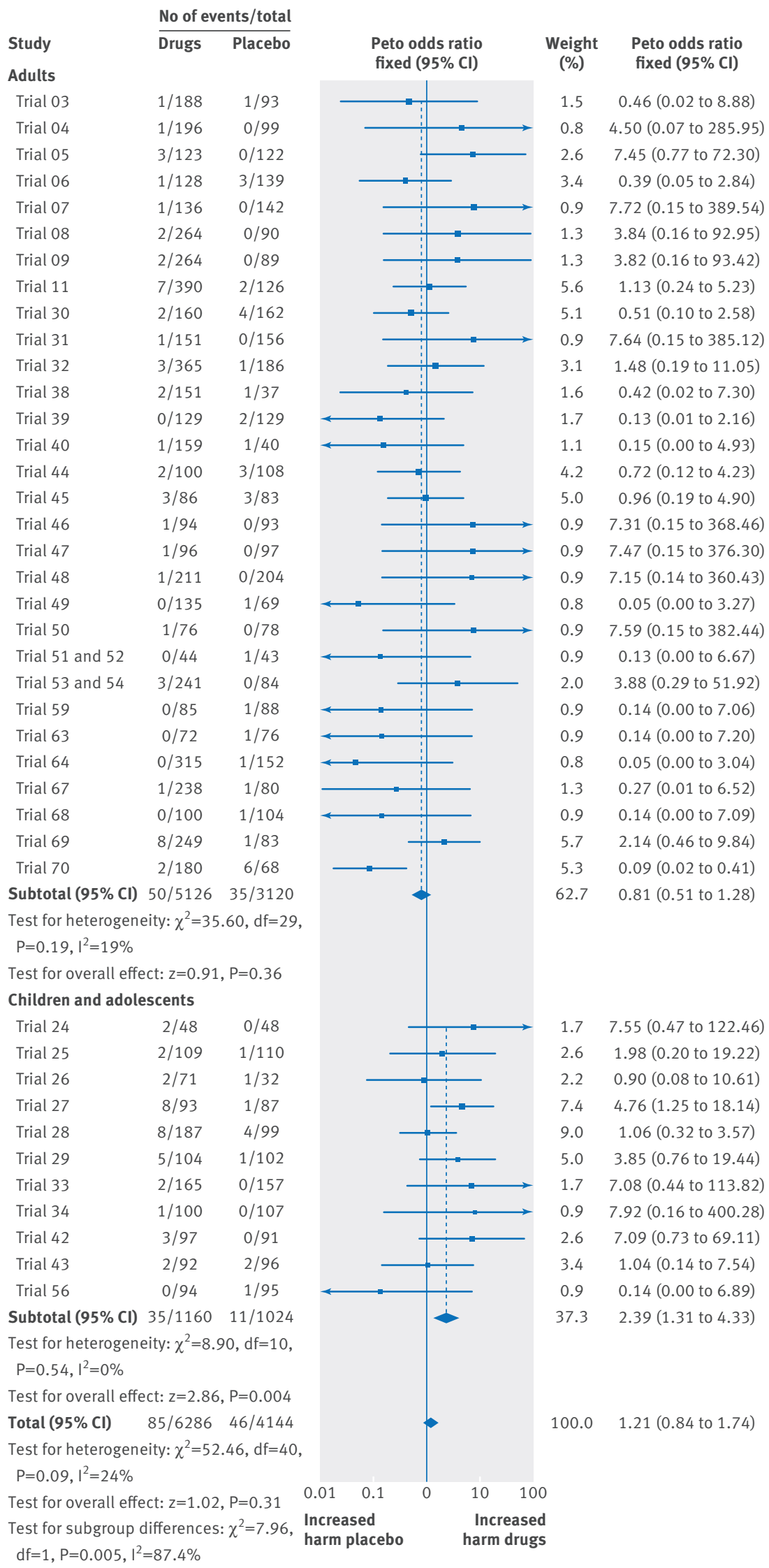

Fig 3 Meta-analysis of suicidality in participants receiving selective serotonin reuptake inhibitors (SSRIs) or serotonin-norepinephrine reuptake inhibitors (SNRIs) compared with placebo post-randomisation going over the appendices containing individual patient listings. This "possible suicide attempt" was listed as "mild" and was not documented elsewhere in the clinical study report and there was no patient narrative.

Five of the 67 post-randomisation events occurred during the lead-out or post-treatment phase of the trials (in three patients receiving study drugs and in two receiving placebo).

Of the remaining 62 suicide attempts (in 59 patients), 40 occurred in 39 patients receiving the study drug, 20 in 18 patients receiving placebo, and two in two patients receiving imipramine. Four of these events were only listed in the individual patient listings and three others only noted in adverse events tables (no further information was available as there was no narrative). Twenty seven events were coded as emotional lability or worsening depression, although in patient narratives or individual patient listings they were clearly suicide attempts. Conversely, several cases of suicidal ideation were called suicide attempts in the adverse events tables. One suicide attempt (intentional overdose with paracetamol (acetaminophen)) in a patient receiving fluoxetine was described as "elevated liver enzymes" in the adverse events tables, in contrast with the narrative (see supplementary data $\mathrm{C}$ ). There was no difference between suicides and suicide attempts (odds ratio 1.05, 95\% confidence interval 0.63 to 1.75 ). The odds ratio for adults was 0.60 (0.29 to 1.24) and for children and adolescents was 1.85 (0.90 to 3.83 , see supplementary data $\mathrm{D}$ ).

\section{Suicidal ideation}

Seventy five participants experienced 76 suicidal ideation events, of which six events were in the lead-in phase (four were taking duloxetine and two placebo). Two of the four events in the duloxetine users were severe and had patient narratives. A third event was mild and was only recorded in treatment emergent adverse events tables. The fourth event, mild suicidal thoughts, appeared only in the appendix containing individual patient listings. Of the 70 post-randomisation events, 41 occurred in participants receiving study drugs, 25 in those receiving placebo, and four in those receiving imipramine.

Sixty two patients experienced 63 events during the randomised phase of the trials (34 events in those receiving drugs, 25 in 24 participants receiving placebo, and four in participants receiving imipramine). Thirty two of these events were coded as emotional lability or worsening of depression in the treatment emergent adverse events tables, but it was clear from the patient narratives or individual patient listings that they were in fact ideation events.

Seven events occurred in the lead-out or post-treatment phases of the trials, and all in participants receiving the study drug (see supplementary data C).

\section{Aggressive behaviour}

Three events of aggressive behaviour in participants receiving duloxetine and two in participants receiving placebo took place before randomisation. Post-randomisation there were 62 events in participants receiving the study drugs, 28 in participants receiving placebo, and four in 


\begin{tabular}{|c|c|c|c|c|c|c|c|c|}
\hline Events & Duloxetine & Fluoxetine & Paroxetine & Sertraline & Venlafaxine & All drugs & Placebo & Imipramine \\
\hline Before randomisation & 3 & 0 & 0 & 0 & 0 & 3 & 2 & 0 \\
\hline Post-randomisation (any arm) & 7 & 6 & 31 & 14 & 4 & 62 & 26 & 4 \\
\hline Total population & 4277 & 456 & 1766 & 3165 & 1263 & 10927 & 6832 & 767 \\
\hline
\end{tabular}

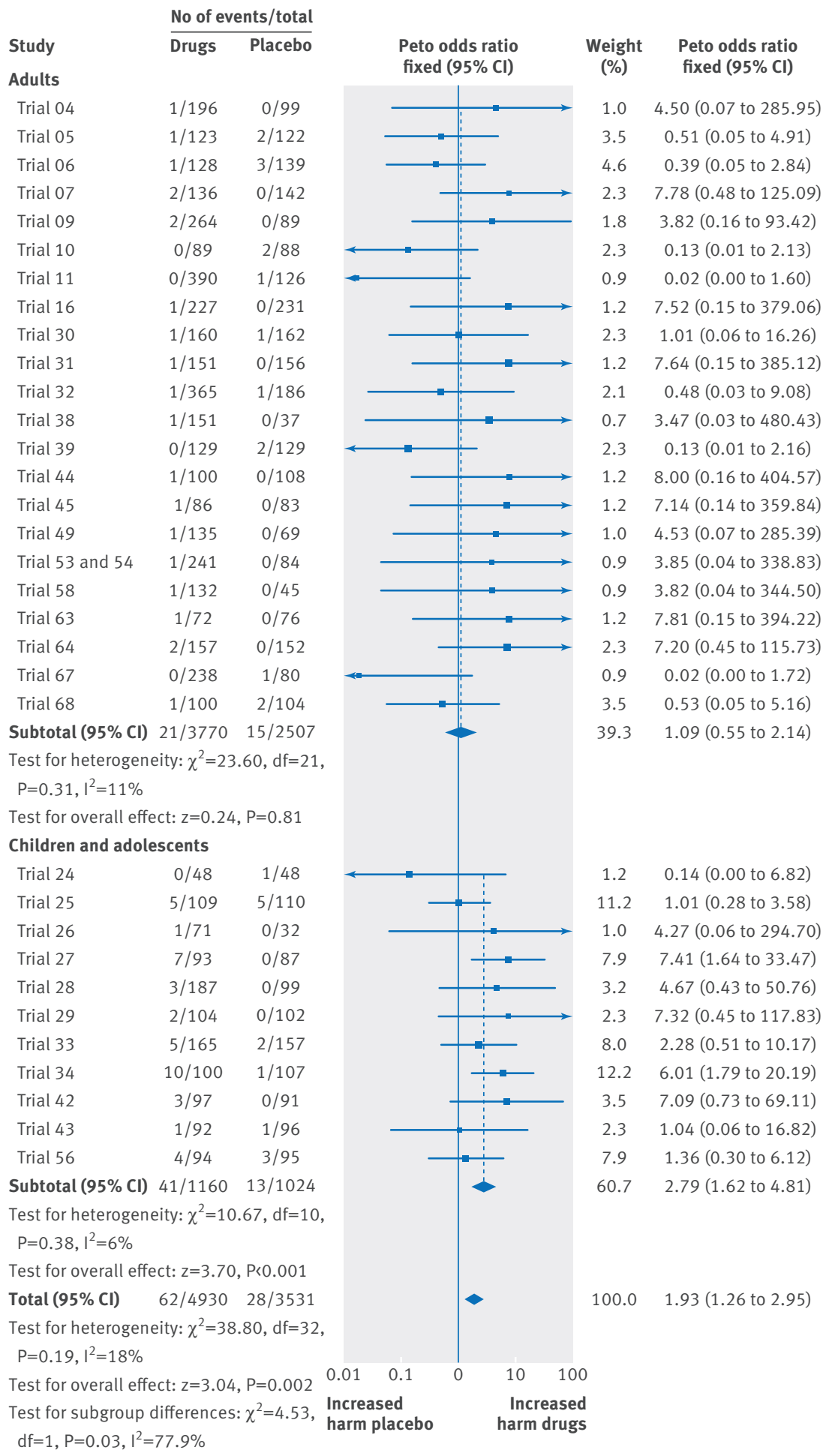

Fig 4 | Meta-analysis of aggressive behaviour in patients receiving selective serotonin reuptake inhibitors (SSRIs) or serotonin-norepinephrine reuptake inhibitors (SNRIs) compared with placebo post-randomisation participants receiving imipramine, of which three in the paroxetine group and two in the placebo group occurred in the lead-out or post-treatment phase (table 4). Aggressive behaviour occurred more often in the drug group compared with placebo group (odds ratio 1.93, 95\% confidence interval 1.26 to 2.95). The odds ratio for adults was 1.09 (0.55 to 2.14) and for children and adolescents was 2.79 (1.62 to 4.81, figure 4). If data were removed from trials 28 and 34 (paediatric trials in which each centre had fraudulent data), the increase in aggression remained: all ages 1.58 (1.00 to 2.51) and children and adolescents only 2.19 (1.17 to 4.11, see supplementary data D).

Only patient narratives were available for serious events and they included homicidal threat, homicidal ideation, assault, sexual molestation, and a threat to take a gun to school (all five participants receiving sertraline), damage to property, punching household items, aggressive assault, verbally abusive and aggressive threats (all five participants receiving paroxetine), and belligerence (fluoxetine). Details were unavailable for non-serious events, as they were either listed in adverse events tables or given in the appendix of individual patient listings without any narratives. These events were increased hostility, aggressiveness, rage, or anger.

\section{Akathisia}

Thirty akathisia events occurred, all post-randomisation (22 in participants receiving study drugs, six in participants receiving placebo, and two in participants receiving clomipramine); two of the events, both in participants receiving duloxetine, took place in the leadout phase (table 5). Akathisia occurred more often in participants receiving the study drug than in those receiving placebo $(2.04,0.93$ to 4.48$)$, but this difference was not statistically significant: for adults 2.00 ( 0.79 to 5.04) and for children and adolescents $(2.15,0.48$ to 9.65 , fig 5). If data were removed from trial 70 (adults), where some centres had fraudulent data, the odds ratio becomes 1.99 ( 0.90 to 4.44) and for adults becomes 1.94 ( 0.75 to 4.99, see supplementary data D).

Some events were not listed as akathisia in the adverse events tables because of the coding dictionaries used. For example, in the three sertraline trials where we had access to both the verbatim and the coded preferred terms, akathisia seemed to have been coded as "hyperkinesia" according to the World Health Organisation Adverse Drug Reaction Terminology dictionary. We could only identify akathisia if we had access to the verbatim terms, which were sometimes available from individual patient listings or patient narratives. For most duloxetine and fluoxetine trials, akathisia was also noted in the regular adverse events tables, and therefore the trials appeared to have more events than those for other drugs for which akathisia 


\begin{tabular}{|c|c|c|c|c|c|c|c|}
\hline Drug (any arm) & Duloxetine & Fluoxetine & Sertraline & Venlafaxine & All drugs & Placebo & Clomipramine \\
\hline Akathisia events & 12 & 7 & 2 & 1 & 22 & 6 & 2 \\
\hline Total population & 4277 & 456 & 3165 & 1263 & 10927 & 6832 & 767 \\
\hline
\end{tabular}

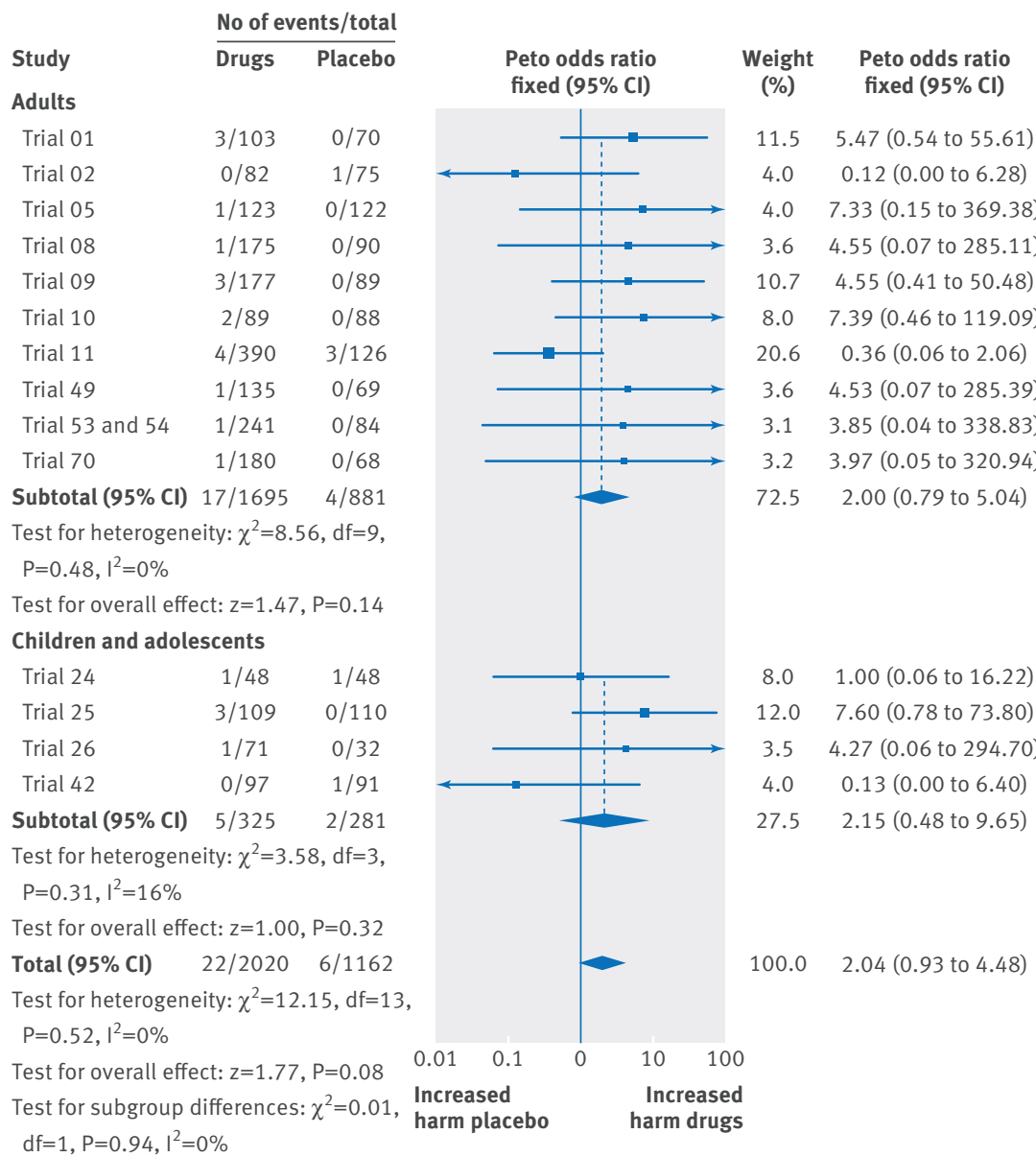

Fig 5 | Meta-analysis of akathisia in participants receiving selective serotonin reuptake inhibitors (SSRIs) or serotonin-norepinephrine reuptake inhibitors (SNRIs) compared with placebo post-randomisation online summary reports for four trials (trials 19-22, all on duloxetine). All the eight deaths (six in participants receiving duloxetine and two in participants receiving placebo) post-randomisation were noted in the online summaries, although information on one suicide in a participant receiving duloxetine in the open label phase before randomisation in trial 7 was missing, as no data from that phase were available online. Only two (both participants receiving fluoxetine) of the 20 suicide attempts (14 participants receiving duloxetine, three fluoxetine, and three placebo) were documented in the summaries, and none of the 14 suicidal ideation events (eight in participants receiving duloxetine, two paroxetine, one fluoxetine, and three placebo) were mentioned. Only 10 (three participants receiving fluoxetine and seven placebo) of the 25 aggressive behaviour events (five participants receiving duloxetine, six fluoxetine, and 14 placebo) were found online. Only three akathisia events (all participants receiving fluoxetine) of the 17 (10 receiving duloxetine, five fluoxetine, and two placebo) were in the summaries. However, the case of the "elevated liver enzymes" in a patient receiving fluoxetine in trial 26 was clarified as an intentional overdose.

\section{Discussion}

Systematic reviews of harms are needed for a balanced view of medical interventions, particularly to elucidate the occurrence of rare but serious events. ${ }^{34}$ Clinical study reports are far more reliable than published trial reports, ${ }^{2428}$ but even using these we were unable to unravel the true number of serious harms. The trials had many shortcomings, in both the design and the reporting of the trials in the clinical study reports, and therefore our numbers are likely to be underestimates. The summary reports on Eli Lilly's website were even more unreliable than we previously suspected. ${ }^{28}$ Only mortality had (almost) complete information. not available. Therefore our number of akathisia events is likely to be an underestimate, as the event appeared to be have been coded under many other activation terms, such as irritability, agitation, or nervousness.

\section{Comparison of our data with the summary trial reports on Eli Lilly's website}

Information was limited on adverse events in these summary reports and it was not reliable. The number of serious events was always mentioned but the cases were not always explained and the reports focused on the most common adverse events. All reports contained tables of treatment emergent adverse events, but not for all patients (with the exception of trials 23 and 26 where complete data were tabulated), and in most cases the events were only shown if they occurred in, for example, at least $5 \%$ of patients. We were unable to find the

\section{Comparison with other studies}

We found no significant differences in mortality or suicidality overall, but our data confirmed the increased risk of suicide in children and adolescents. ${ }^{516}$ We wanted to clarify these risks in adults and found no significant increase in association with drugs, similar to previous analyses. $^{78}$ Our results however, cannot be compared easily with the results of the 2006 FDA meta-analysis $^{7}$ as we had data from 18526 patients, whereas the FDA included about 100000 patients. The FDA did not consider the limitations of the trials that we identified and introduced some of their own-for example, by only phase was over. We counted all post-randomisation events in our study, although they were not always available. Interestingly, an FDA employee published a counting events within 24 hours after the randomised 
paper in 2001 using FDA data that showed 22 suicides in 22062 patients randomised to antidepressants, ${ }^{35}$ which equates to 10 per 10000 population, but in the large FDA meta-analysis five years later, five suicides were reported in 52960 patients, or 1 per 10000 population. $^{7}$

A review with over 40000 patients using data submitted to the UK's Medicines and Healthcare products Regulatory Agency (MHRA) also found no increased risk for suicidality in adults using serotonin reuptake inhibitors (SSRIs), but noted that the relative frequency of reported self harm and suicidal thoughts in the trials compared with suicide indicated that non-fatal endpoints were under-recorded. ${ }^{8}$ Another review, with 87650 patients (all ages), reported a doubling in the odds of suicide attempts, which was statistically significant, ${ }^{1}$ in contrast with our findings in adults. As with our study, both reviews found serious limitations in the trials and evidence of under-reporting of serious harms.

This under-reporting was also confirmed in the recent republication by independent investigators of study 329 of paroxetine in children and adolescents. ${ }^{36}$ We did not get access to the appendices of this trial, which contained the individual patient listings. Many suicidal events were only documented there, and even more suicidal events were only identified in the case report forms, which the investigators got access to after protracted negotiations with GlaxoSmithKline and then only through a single screen remote desktop interface, which made it impossible for the researchers to review all 77000 pages. $^{36}$

We found that the risk of aggressive behaviour was doubled with use of antidepressants (all ages), which was a statistically significant result, but when we restricted our analysis to adults, there was no such effect. However, we did find a doubling of risk for children and adolescents, which is consistent with the increased incidence in hostility noted by the MHRA. ${ }^{16}$ We found that akathisia was much under-reported. Akathisia occurred more often in participants receiving drugs than receiving placebo, both in children and adolescents and in adults, but the difference was not significant (all ages, odds ratio $2.04,95 \%$ confidence interval 0.93 to 4.48 ). We also found similar results in a systematic review of trials in healthy adult volunteers that included data from 10 published trials and two unpublished trials (clinical study reports obtained from EMA). Compared with placebo $(n=226)$, antidepressants $(n=318)$ were associated with an increased rate of activation or other precursor events for aggression and suicidality (odds ratio 1.81, 95\% confidence interval 1.05 to 3.12$).{ }^{37}$

Limitations in the trials and clinical study reports In most trials $(86 \%)$, patients were only randomised if they failed to improve in the placebo lead-in period. One large trial had a 12 week open label period where 533 patients received duloxetine and only 278 patients (52\%) who tolerated the drug were randomised. This gives rise to response based selection bias, which has an impact on the subsequent randomised phase. During that open label period for duloxetine, there was one suicide (by hanging), four suicide attempts, and four suicidal ideation events.
Another problem was insufficient lead-in periods. ${ }^{424}$ At least 36 trials had insufficient wash-out periods, lasting for only a few days or a week. An additional nine trials had no lead-in period. Even when a placebo lead-in period was specified it was not always adhered to-for example, in a venlafaxine trial (trial 70), the wash-out period was inadequate in 30 patients who received drugs before the study, and in a sertraline trial (trial 50) it was stated that "some patients proceeded to double-blind treatment without a prior placebo run-in.” As patients are often receiving treatment with similar drugs already, some may develop withdrawal effects when they are switched to a placebo, ${ }^{2412142324}$ which can be wrongly counted as adverse events. These iatrogenic harms can be substantial. In a large study supported by Eli Lilly, withdrawal symptoms were registered in patients during a 5-8 day period; 4-24 months after their depression had remitted. Placebo was substituted for active drug, unknown to the patients, and when the patients were switched to placebo, about one third receiving sertraline or paroxetine became agitated, irritable, reported worsened mood, and their Hamilton depression score increased by at least $8 .{ }^{38}$

Most trials did not report on post-treatment events. As previously noted, the FDA included events occurring within the first 24 hours after the randomised phase ended. ${ }^{7}$ For sertraline trials in adults (the report's table 30; we reanalysed this summary data), there was no increased risk of suicide or suicide attempts (risk ratio 0.87, 95\% confidence interval 0.31 to 2.48). ${ }^{7}$ When Pfizer analysed its trial data, the results looked much better for sertraline (we reanalysed their data for suicide or suicide attempts); risk ratio 0.52 (0.17 to 1.59). ${ }^{39}$ However, Pfizer published an additional analysis where the patients were followed up for 30 days after the randomised phase ended and then sertraline did not seem to protect against suicides or suicide attempts in adults but rather seemed to cause them (we reanalysed their data, risk ratio 1.47, 0.77 to 2.83), even though these findings were not significant. ${ }^{39}$ The investigators who used MHRA data ${ }^{8}$ found that when events after 24 hours were included, the risk of suicide or self harm was doubled with sertraline: we reanalysed the data (risk ratio 2.14, 0.96 to 4.75), although the finding was not statistically significant (see supplementary data D). ${ }^{7}$

Another limitation was the use of different coding dictionaries; 32 trials (46\%) did not state which one they used. Sixteen of the sertraline trials used the World Health Organisation Adverse Drug Reaction Terminology, and as it does not allow for coding of akathisia or suicidal ideation, such events are most likely to be underestimated in our review. Furthermore, we found that many suicidal ideation events were coded as "worsening depression" or "emotional lability" in treatment emergent adverse events tables in the paroxetine trials, which used their own dictionary (the Adverse Drug Experience Coding System, ADECS), as has been noted by other studies. ${ }^{3640}$ Only one trial (trial 27) mentioned this problem in the clinical study report, which stated that "emotional lability captures events such as suicidal ideation/gestures as well as overdoses.” We 
could not find any akathisia events in the paroxetine trials, as we did not have access to the verbatim terms and the events were coded as other activation terms despite akathisia being the preferred term in the Coding Symbols for a Thesaurus of Adverse Reaction Terms dictionary, on which ADECS is based. ${ }^{41}$

Minor tranquillisers and sleeping aids were used in many of the studies, which tend to obscure aggression and akathisia events. Additionally, two thirds of all trials excluded patients at risk of suicide.

\section{Strengths and limitations of this review}

We believe ours is the first comprehensive review of randomised controlled trial data using clinical study reports for aggressive behaviour and akathisia, and our finding of the doubling of aggression in children and adolescents is novel. Our review has highlighted limitations in the trials, not only in their design but also in their reporting in the clinical study reports, which may have led to serious under-estimation of the harms.

A main limitation of our review was that the quality of the clinical study reports differed vastly and ranged from summary reports to full reports with appendices, which limited our ability to detect the harms. Our study also showed that the standard risk of bias assessment tool was insufficient when harms from antidepressants were being assessed in clinical study reports. Most of the trials excluded patients with suicidal risk and so our numbers of suicidality might be underestimates compared with what we would expect in clinical practice. We also did not have access to case report forms and because of coding problems we deliberately took a conservative approach and used only one term for identifying akathisia.

\section{Conclusions and implications for research and practice}

We believe our study shows that, despite using clinical study reports, the true risk for serious harms is still uncertain. The low incidence of these rare events and the poor design and reporting of the trials makes it difficult to get accurate effect estimates.

The FDA has advised that antidepressants may also cause suicide in young adults (18 to 24 years) and recommends that "patients of all ages" treated with antidepressants should be monitored for "clinical worsening, suicidality, and unusual changes in behaviour." 42 GlaxoSmithKline also issued letters to doctors, informing them about the increased harm in young adults ${ }^{6}$ and admitted that for adults with depression "(all ages), the frequency of suicidal behaviour was higher in patients treated with paroxetine compared with placebo: $11 / 3455$ $(0.32 \%)$ versus 1/1978 (0.05\%).”43 A cohort study from Sweden recently showed an increase in violent crime in young adults taking antidepressants (hazard ratio 1.43, 95\% confidence interval 1.19 to 1.73$) .44$

Therefore we suggest minimal use of antidepressants in children, adolescents, and young adults, as the serious harms seem to be greater, and as their effect seems to be below what is clinically relevant. ${ }^{445-47}$ Alternative treatments such as exercise $\mathrm{e}^{4849}$ or psychotherapy ${ }^{450}$ may have some benefit and could be considered, although psychotherapy trials also suffer from publication bias..$^{51}$

The need for identifying hidden information in clinical study reports to form a more accurate view of the benefits and harms of drugs has been highlighted by the Restoring Invisible and Abandoned Trials (RIAT) initiative, ${ }^{52}$ and the recent revised version of trial $329 .{ }^{36}$ More data from clinical study reports are expected to become available in the coming years, with the EMA's new policy to make all newly submitted reports publicly available. ${ }^{53}$ As it can be quite labour intensive to perform systematic reviews using clinical study reports, more reliable automated methods for text mining are needed, such that all data, including that from individual patient listings and case report forms, can be routinely considered. ${ }^{3654}$

We thank the European Medicines Agency and Medicines and Healthcare products Regulatory Agency for providing the clinical study reports used for this review. Some results of this study were presented at the Research waste/EQUATOR conference in Edinburgh, Scotland (September 2015)

Contributors: All authors had complete access to the data in the study. LSG was known by her maiden name Jensen at the time of the study. TS and PCG contributed to the study concept and design, wrote the protocol, and obtained funding. TS, LSG, and NF acquired the data for the study; all authors contributed to the analysis and/or interpretation of data. TS developed the first draft of the manuscript and all authors critically revised the manuscript and approved the final version. PCG is the study supervisor and guarantor.

Funding: This study is part of a PhD (TS) thesis, funded by the Laura and John Arnold Foundation. The funding source had no role in the design and conduct of the study; data collection, management, analysis, and interpretation; preparation, review, and approval of the manuscript; or the decision to submit the paper for publication.

Competing interests: All authors have completed the ICMJE uniform disclosure form at http://www.icmje.org/coi disclosure.pdf (available on request from the corresponding author) and declare: this study is part of a PhD funded by the Laura and John Arnold Foundation for lead author (TS); no financial relationships with any organisations that might have an interest in the submitted work in the previous three years; no other relationships or activities that could appear to have influenced the submitted work.

Ethical approval: Not required.

Transparency: The lead author (TS) and study guarantor (PCG) affirm that the manuscript is an honest, accurate, and transparent account of the study being reported. No important aspect of the study has been omitted. No discrepancies are withheld.

Data sharing: Additional data and the clinical study reports can be obtained from the corresponding author on request.

This is an Open Access article distributed in accordance with the Creative Commons Attribution Non Commercial (CC BY-NC 3.0) license, which permits others to distribute, remix, adapt, build upon this work non-commercially, and license their derivative works on different terms, provided the original work is properly cited and the use is non-commercial. See: http://creativecommons.org/licenses/ by-nc/3.0/

1 Fergusson D, Doucette S, Glass KC, et al. Association between suicide attempts and selective serotonin reuptake inhibitors: systematic review of randomised controlled trials. BM/ 2005;330: 396. doi:10.1136/bmj.330.7488.396

Healy D. Let them eat Prozac. New York University Press, 2004

3 Teicher MH, Glod C, Cole JO. Emergence of intense suicidal preoccupation during fluoxetine treatment. Am J Psychiatry 1990;147: 207-10. doi:10.1176/ajp.147.2.207

4 Gøtzsche PC. Deadly psychiatry and organised denial.People’s Press, 2015.

5 Whittington CJ, Kendall T, Fonagy P, et al. Selective serotonin reuptake inhibitors in childhood depression: systematic review of published versus unpublished data. Lancet 2004;363: 1341-5.

6 Healy D. Did regulators fail over selective serotonin reuptake inhibitors?BM/ 2006:333: 92-5. doi:10.1136/bmj.333.7558.92 Laughren TP. Overview for December 13 meeting of Psychopharmacologic Drugs Advisory Committee (PDAC). 2006 www.fda.gov/ohrms/dockets/ac/06/briefing/2006-4272b1-01-fda. pdf. 
8 Gunnell D, Saperia J, Ashby D. Selective serotonin reuptake inhibitors (SSRIs) and suicide in adults: meta-analysis of drug company data from placebo controlled, randomised controlled trials submitted to the MHRA's safety review. BM/ 2005;330: 385. doi:10.1136/bmj.330.7488.385

9 Bond AJ. Antidepressant treatments and human aggression. Eur J Pharmacol 2005:526: 218-25. doi:10.1016/j.ejphar.2005.09.033

10 Bouvy PF, Liem M. Antidepressants and lethal violence in the Netherlands 1994-2008. Psychopharmacology (Berl) 2012;222: 499-506. doi:10.1007/s00213-012-2668-2

11 Breggin PR. Fluvoxamine as a cause of stimulation, mania, and aggression: a critical analysis of the FDA-approved label. Ethical Hum SciServ 2002; 4: 211-27

12 Breggin PR. Suicidality, violence and mania caused by selective serotonin reuptake inhibitors (SSRIs): a review and analysis. Int J Risk SafMed 2003;16: 31-49

13 Krakowski M. Violence and serotonin: influence of impulse control, affect regulation, and social functioning. I Neuropsychiatry Clin Neurosci 2003;15: 294-305. doi:10.1176/jnp.15.3.294

14 Lucire Y, Crotty C. Antidepressant-induced akathisia-related homicides associated with diminishing mutations in metabolizing genes of the CYP450 family. Pharmgenomics Pers Med 2011;4: 65-81. doi:10.2147/PGPM.S17445

15 Walsh MT, Dinan TG. Selective serotonin reuptake inhibitors and violence: a review of the available evidence. Acta Psychiatr Scand 2001;104: 84-91. doi:10.1034/j.1600-0447.2001.00357.x

16 United Kingdom Committee on Safety of Medicines, CSM. report of the CSM Expert Working Group on the safety of selective serotonin reuptake inhibitor antidepressants. Medicines and Healthcare products Regulatory Agency; 2004

17 Moore TJ, Glenmullen J, Furberg CD. Prescription drugs associated with reports of violence towards others. PLoS One 2010;5: e15337. doi:10.1371/journal.pone.0015337

18 SSRI stories. SSRI stories: antidepressant nightmares. 2013. http:// ssristories.org/.

19 Adler LA, Angrist BM. Paroxetine and akathisia. Biol Psychiatry 1995;37: 336-7. doi:10.1016/0006-3223(94)00158-Y

20 Baldassano CF, Truman C), Nierenberg A, Ghaemi SN, Sachs GS. Akathisia: a review and case report following paroxetine treatment. Compr Psychiatry 1996;37: 122-4. doi:10.1016/S0010-440X(96)90572-6.

21 Bass A. Side effects: a prosecutor, a whistleblower, and a bestselling antidepressant on trial.Algonquin Books, 2008.

22 Breggin P, Breggin GR. Talking back to Prozac: what doctors aren't telling you about today's most controversial drug. E-reads, 1994.

23 Breggin P. Medication madness: the role of psychiatric drugs in cases of violence, suicide, and crime.St Martin's Griffin, 2008.

24 Healy D, Herxheimer A, Menkes DB. Antidepressants and violence: problems at the interface of medicine and law. PLoS Med 2006;3: e372. doi:10.1371/journal.pmed.0030372

25 Miller M, Swanson SA, Azrael D, Pate V, Stürmer T. Antidepressant dose, age, and the risk of deliberate self-harm. JAMA Intern Med 2014;174: 899-909. doi:10.1001/jamainternmed.2014.1053

26 Koliscak LP, Makela EH. Selective serotonin reuptake inhibitorinduced akathisia. J Am Pharm Assoc (2003) 2009;49: e28-36, quiz e37-8. doi:10.1331/JAPhA.2009.08083

27 Wieseler B, Wolfram N, McGauran N, et al. Completeness of reporting of patient-relevant clinical trial outcomes: comparison of unpublished clinical study reports with publicly available data. PLoS Med 2013;10:e1001526.

28 Maund E, Tendal B, Hróbjartsson A, et al. Benefits and harms in clinical trials of duloxetine for treatment of major depressive disorder: comparison of clinical study reports, trial registries, and publications. BM/ 2014:348: g3510. doi:10.1136/bmi.g3510

29 Stone M, Laughren T, Jones ML, et al. Risk of suicidality in clinical trials of antidepressants in adults: analysis of proprietary data submitted to US Food and Drug Administration. BMJ 2009;339: b2880. doi:10.1136/bmi.b2880

30 Lilly E. Clinical Trial Report (CTR) summaries. 2014. www.lillytrials. com/results/ctr_toc.pdf.

31 Higgins JPT, Green S, eds. Cochrane handbook for systematic reviews of interventions version 5.1.0. Cochrane Collaboration, 2011. www. cochrane-handbook.org

32 Sweeting MJ, Sutton AJ, Lambert PC. What to add to nothing? Use and avoidance of continuity corrections in meta-analysis of sparse data. StatMed 2004;23: 1351-75. doi:10.1002/sim.1761

33 Structure and content of clinical study reports: E3. International Conference on Harmonisation of Technical Requirements for Registration of Pharmaceuticals for Human Use, 1995. www.ich.org/ fileadmin/Public_Web_Site/ICH_Products/Guidelines/Efficacy/E3/ E3 Guideline.pdf
34 Chou R, Helfand M. Challenges in systematic reviews that assess treatment harms. Ann Intern Med 2005;142(12_Part_2):1090-9.

35 Laughren TP. The scientific and ethical basis for placebo-controlled trials in depression and schizophrenia: an FDA perspective. Eur Psychiatry 2001;16: 418-23. doi:10.1016/S0924-9338(01)00600-9

36 Le Noury J, Nardo JM, Healy D, et al. Restoring Study 329: efficacy and harms of paroxetine and imipramine in treatment of major depression in adolescence. BMJ 2015;351: h4320. doi:10.1136/bmj.h4320

37 Bielefeldt A $\varnothing$, Danborg PB, Gøtzsche PC. Systematic review of adverse effects of antidepressants in healthy volunteer studies. Proceedings of the 23rd Cochrane colloquium. Vienna, Austria; Cochrane 2015.

38 Rosenbaum JF, Fava M, Hoog SL, Ascroft RC, Krebs WB. Selective serotonin reuptake inhibitor discontinuation syndrome: a randomized clinical trial. Biol Psychiatry 1998;44: 77-87. doi:10.1016/ S0006-3223(98)00126-7

39 Vanderburg DG, Batzar E, Fogel I, Kremer CM. A pooled analysis of suicidality in double-blind, placebo-controlled studies of sertraline in adults. J Clin Psychiatry 2009;70: 674-83. doi:10.4088/JCP.07m04004

40 Furukawa TA. All clinical trials must be reported in detail and made publicly available. BMJ 2004;329: 626. doi:10.1136/ bmj.329.7466.626-a

41 Breggin PR. How GlaxoSmithKline suppressed data on Paxil-induced akathisia: implications for suicidality and violence. Ethical Hum Psychol Psychiatry 2006;8: 91-100.doi:10.1891/ehpp.8.2.91.

42 FDA. Antidepressant use in children, adolescents, and adults. 2014. www.fda.gov/downloads/Drugs/DrugSafety/ InformationbyDrugClass/UCM173233.pdf.

43 GlaxoSmithKline. Important prescribing information. Letter to healthcare professionals, May 2006. www.gsk.com/media/388699/ adult_hcp_letter.pdf.

44 Molero Y, Lichtenstein P, Zetterqvist J, Gumpert CH, Fazel S. Selective serotonin reuptake inhibitors and violent crime: a cohort study. PLOS Med 2015;12: e1001875. doi:10.1371/journal.pmed.1001875

45 Gøtzsche PC, Young AH, Crace J. Does long term use of psychiatric drugs cause more harm than good?BMJ 2015;350: h2435. doi:10.1136/bmi.h2435

46 Leucht S, Fennema H, Engel R, Kaspers-Janssen M, Lepping P, Szeged A. What does the HAMD mean? Jffect Disord 2013;148: 243-8. doi:10.1016/j.jad.2012.12.001

47 MoncrieffJ, Wessely S, Hardy R. Active placebos versus antidepressants for depression. Cochrane Database Syst Rev 2004;1: CD003012.

48 Larun L, Nordheim LV, Ekeland E, Hagen KB, Heian F. Exercise in prevention and treatment of anxiety and depression among children and young people. Cochrane Database Syst Rev 2006;3: CD004691.

49 Cooney GM, Dwan K, Greig CA, et al. Exercise for depression. Cochrane Database Syst Rev 2013;9: CD004366.

50 Cox GR, Callahan P, Churchill R, et al. Psychological therapies versus antidepressant medication, alone and in combination for depression in children and adolescents. Cochrane Database Syst Rev 2012;11: CD008324.

51 Driessen E, Hollon SD, Bockting CL, Cuijpers P, Turner EH. Does Publication bias inflate the apparent Efficacy of psychological treatment for major depressive disorder? A systematic review and meta-analysis of US National Institutes of Health-funded trials. PLOS One 2015;10: e0137864. doi:10.1371/journal.pone.0137864

52 Doshi P, Dickersin K, Healy D, Vedula SS, Jefferson T. Restoring invisible and abandoned trials: a call for people to publish the findings. BMJ 2013;346: f2865. doi:10.1136/bmj.f2865

53 European Medicines Agency. Publication and access to clinical-tria data. 2014.www.ema.europa.eu/docs/en_GB/document_library/ Other/2013/06/WC500144730.pdf.

54 Cohen D. Rosiglitazone: what went wrong?BMJ 2010;341: c4848. doi:10.1136/bmj.c4848

\section{Web extra material}

Supplementary data A: additional details on methods Supplementary data B: trial characteristics of included 70 randomised controlled trials

Supplementary data C: case notes for primary outcomes Supplementary data D: additional analyses 